Eine kulturhistorische Studie zu den Neologismen in Stefan Zweigs Schachnovelleanhand von Viktor Klemperers Buch LTI, Notizbuch eines Philologen در اسة حضارية تاريخية حول التجديدات اللغوية فى رواية "لاعبوا الشطرنج" للكاتب "شتيفان تسفايج" وتحليلها من خلال رؤ رؤى الته الناقد فيكتوركليمبرر فى كتابه "مفكرة أحد علماء اللغة تلفة

Dr. Ibrahim Hamed Abdella

Assisstant Professor - Abteilung für Germanistik Philosophische Fakultät der Beni Souef Universität

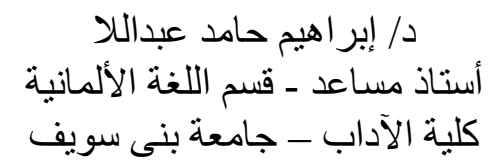



Summary:

A colloquium on "Transcultural Society" took place in Cairo from 12 to 15 March 2015. To this end, I wrote a work entitled "Transcultural Society and Identity with the example of Stefan Zweig's Schachnovelle". When working on this subject, I found out how differently this novella is to be interpreted, and how many perspectives this work can still be considered profitable today. Although a great deal of scientific work has already been written about it, one can still gain new insights from this work. Therefore, the present work is a supplement to my first work, and I intend to add a second supplement to it. In this thesis the linguistic changes of the German language and their precipitation in the Schachnovelle by Stefan Zweig (1881-1942) will be discussed. For example, it should be shown how the history of the cultural history in the Austrian Empire has developed and whether this development can be demonstrated by Stefan Zweig.

The timeless relevance of the chess novella is also due to linguistic historical retreats. In the course of action, the events are described as distant as in a scientific work. For example, Dr. B. tells his traumatic experiences and his feelings as precisely as if Sigmund Freud had personally received them. It is the writing style of naturalism, which also draws on historical formulations to be described as historical neologisms. These are to be shown by means of examples in this work. 


\section{Eine kulturhistorische Studie zu den Neologismen in Stefan Zweigs Schachnovelle anhand von Viktor Klemperers Buch LTI, Notizbuch eines Philologen}

\section{Einführung}

In Kairo fand vom 12.-15.03.2015 ein Kolloquium zum Thema „Transkulturelle Gesellschaft“ statt. Dazu schrieb ich eine Arbeit mit dem Titel „Transkulturelle Gesellschaft und Identität am Beispiel von Stefan Zweigs Schachnovelle". Bei der Bearbeitung dieses Stoffes stellte ich fest, wie differenziert diese Novelle zu interpretieren ist, und aus wie vielen Perspektiven man dieses Werk noch heute gewinnbringend betrachten kann. Denn obwohl schon sehr viele wissenschaftliche Arbeiten darüber verfasst wurden, kann man aus diesem Werk immer noch neue Erkenntnisse gewinnen. Deshalb ist die vorliegende Arbeit ein Supplement meiner ersten Arbeit, und ich beabsichtige, dem noch ein zweites weiteres Supplement hinzuzufügen. In dieser Arbeit soll es um die sprachlichen Veränderungen der deutschen Sprache und deren Niederschlag in der Schachnovelle von Stefan Zweig (1881-1942) gehen. Zum Beispiel soll dargelegt werden, wie die kulturhistorische Sprachentwicklung im österreichischen Kaiserreich verlaufen ist und ob diese Entwicklung bei Stefan Zweig nachgewiesen werden kann.

Die zeitlose Relevanz der Schachnovelle ist auch den sprachlichen historischen Rückgriffen geschuldet. Denn in dem Handlungsstrang werden die Ereignisse so distanziert geschildert wie in einer wissenschaftlichen Arbeit. Zum Beispiel erzählt Dr. B. seine traumatischen Erlebnisse und seine Gefühle so präzise, als hätte sie Sigmund Freud persönlich rezipiert. Es ist der Schreibstil des Naturalismus, der auch auf historische Formulierungen zurückgreift, die als historistische Neologismen zu beschreiben sind. Diese sollen anhand von Beispielen in dieser Arbeit aufgezeigt werden.

Stefan Zweig musste schon sehr bald in seinem Leben Bekanntschaft mit dem Antisemitismus machen. Um diese Entwicklung heute noch zu verstehen, kann die Lektüre von Victor Klemperer LTI Notizbuch eines Philologen eine Anleitung sein. Denn Klemperer befasst sich mit der Sprache In Tempore Nazi, also mit der Art der Sprache, wie sie den Menschen während der Zeit des Nationalsozialismus durch die 
NS-Propaganda - wieder und wieder eingehämmert wurde. Dass der Titel LTI eine Parodie ist, geht daraus hervor, dass Klemperer selbst eine Abbreviatur verwendet. Denn in der Langform heißt der Titel Lingua Tertii Imperii, und der lateinische Titel steht für „Sprache des Dritten Reiches". In der vorliegenden Arbeit soll an dem Beispielwort fanatisch verdeutlicht werden, wie nahe die psychiatrische Wissenschaftssprache, die Literatursprache und psychiatrische Neologismen sowie die Amtssprache des sog. „Hitlerdeutsch“ (ein von Victor Klemperer verwendeter Ausdruck) beieinander lagen. Wie der Rassismus und die nazistische Sprache entstanden sind, und welche Wurzeln sie hatten, dem soll in diesem Kapitel der Arbeit nachgegangen werden. Denn die Notwendigkeit, eigene Neologismen zu entwickeln, begann für Stefan Zweig schon frühzeitig, als er im Kaiserreich Österreich unheilvolle Bekanntschaft mit der antisemitischen Politikrichtung machte. Denn die Antisemiten, welche die Denkweise von Adolf Hitler beeinflussten, kamen aus Österreich. Die antisemitische Ausdrucksweise soll in dieser Arbeit an Hand von Beispielen, auch von Victor Klemperer, erläutert werden. Dabei sollen einflussreiche und zum Teil mächtige Antisemiten sowie der spätere Diktator, der damals noch junge Adolf Hitler, genannt und zitiert werden.

Ob der Einfluss dieser Leute der nazistischen Sprache zum Durchbruch verhalf, oder ob es auch noch andere bekannte Schreiber gab, die sich als geistige Brandstifter hervortaten und dem Nationalsozialismus Vorschub leisteten, soll anschließend untersucht werden. Welche Einflüsse es außerdem in Deutschland gab, die das Entstehen der nazistischen Sprache begünstigten, sollen dargelegt werden.

Victor Klemperer geht der Wurzel der Sprache des deutschen Antisemitismus nach und bezeichnet dabei das Werk Partenau von Max René Hesse als „Vorwegnahme der Sprache der Gesinnungen des Dritten Reichs“. Er diagnostizierte den deutschen Hang zur Romantik, als die „Wurzel der nazistischen Sprache“. Auch diese Zusammenhänge sollen in dieser Arbeit beschrieben werden. ${ }^{1}$

Der damalige Wissensstand in der Philosophie wurde vom NSRegime in Frage gestellt und durch eine nazistische Weltanschauung ersetzt. $\mathrm{Ob}$ es dafür einen Niederschlag in Form von neologischen 
Beispielen in der Schachnovelle gibt, soll untersucht werden. Das Adjektiv fanatisch führt in dieser Arbeit in verschiedenen Auslegungssituationen über Sigmund Freud, Stefan Zweig zu einer Anekdote von Victor Klemperer. In diesem Kapitel der vorliegenden Arbeit sollen die verschiedenen Auslegungen aufgelistet und die Frage geklärt werden, ob fanatisch eventuell auch ein psychiatrischer Neologismus ist.

Stefan Zweig schrieb unter dem Einfluss von Sigmund Freud Novellen, die von Freud psychoanalytisch gedeutet und bewertet wurden. Stefan Zweig bekannte in einem Brief an Freud, dass die Psychologie die eigentliche Passion seines Lebens sei. Thomas Anz schrieb in Verwirrung der Gefühle. ${ }^{2}$ Stefan Zweig und Sigmund Freud über den Briefwechsel zwischen Freud und Zweig: Stefan Zweigs Novellen wurden von Freud gelesen, und Freud schrieb 1908 an Zweig: „In Ihre Gedanken finde ich mich leicht hinein als wären es meine guten Bekannten". ${ }^{3}$

Also bewunderte Freud Stefan Zweig im Bereich der psychologischen Literatur, und Zweig bewunderte Sigmund Freud im Bereich der wissenschaftlichen Psychologie. In dieser Arbeit soll am Beispiel des Adjektivs fanatisch versucht werden herauszufinden, ob sich dafür in der Schachnovelle Anhaltspunkte finden lassen.

Anschließend soll der Sprachstil des NS-Regimes auf linguistische Sprachverkürzungen, d. h. auf Abbreviaturen hin untersucht werden. Denn auch Stefan Zweig verwendete den Begriff der Abbreviatur als sprachliche Abwehrstrategie gegen die Hybris eines imperialistischen Schach-Weltmeisters.

Stefan Zweig beherrschte u. a. die französische Sprache perfekt in Wort und Schrift. Denn Französisch war die führende Sprache in Europa schon seit der Zeit der Renaissance, als Frankreich zwischen 1494 und 1559 eine Reihe von Kriegen gegen Italien führte. Damit begann die Transformation aus dem lateinisch-italienischen Sprachraum nach Frankreich und später in den europäischen Sprachraum. Die vorliegende Arbeit soll einige der von Stefan Zweig verwendeten französischen Neologismen in der Schachnovelle aufzeigen. 
In der Bildungsgeschichte war die Sprache, mit der Bildung vermittelt wurde, nicht immer nur die Muttersprache, sondern auch Fremdsprachen. Beispielsweise galt den Römern im Imperium Romanum die griechische Sprache als Ausdruck von Bildung und Gelehrtheit. Diese Tradition wurde bis in die frühe Neuzeit, d.h. bis zum Übergang vom 18 . zum 19. Jahrhundert, gepflegt, also auch noch zurzeit von Stefan Zweig.Welchen Nutzen der Dichter aus der Kenntnis der alt-griechischen Sprache zog, und ob er sie als Neologismen einsetzte, um nicht in die Nähe der hitlerdeutschen Ausdrucksweise zu geraten, ist Teil dieser Arbeit.

In Westeuropa war Latein vom Mittelalter bis in die frühe Neuzeit die allgemeine Bildungssprache und die Sprache der Wissenschaften und deshalb auch für Stefan Zweig ab seinem Eintritt ins Gymnasium ein Schulfach, dass ihm bis zu seiner Matura erhalten blieb. Denn Latein war bis ins 19. Jahrhundert die Sprache der akademischen Berufe in dieser alten Sprache wurden bis ins 20. Jahrhundert hinein noch wissenschaftliche Abhandlungen verfasst.

\section{Die Wurzeln der deutschen Sprachentwicklung}

Der erste Teil der kulturellen Prägung von Stefan Zweig ist der im österreichischen Kaiserreich bis ca. 1750 im offiziellen Schriftstücken verwendeten oberdeutschen Schreibsprache geschuldet. Diese hat einige Besonderheiten, sowohl in der Rechtschreibung als auch vor allem im Vokabular und in der Idiomatik.

Als Beispiel gibt es in der Schachnovelle den Ausdruck Gehaben

„[...] und seiner mühsam manikürten Finger blieb er in seinem Gehaben und seinen Manieren derselbe beschränkte Bauernjunge, der und seinen Manieren derselbe beschränkte Bauernjunge, der im Dorf die Stube des Pfarrers gefegt. ${ }^{\text {(4 }}$

Gehaben ist in der hochdeutschen Sprache ein regelwidriger Begriff, denn heute spricht man von dem aus dem Mittelhochdeutschen stammenden Wort von Gehabe ohne „n“. Das Wort beschreibt im weitesten Sinne das Benehmen, ist aber nur als ein Singularetantum bekannt. Der Wortstamm von Gehaben kommt augenfällig aus dem Lateinischen habere, das haben bedeutet, zum Beispiel: Benehmen haben. 
Im Oberdeutschen ist Gehaben ein Reciprocum, das heißt, es ist ein Verhalten auf Gegenseitigkeit. Ergo bedeutet Gehaben im österreichischen Sprachraum so viel wie die Art und Weise sich zu benehmen, das in die oberdeutsche Schreibsprache adaptiert wurde.

Ein anderes Beispiel ist das Verb ,hinrief":

,[...] wo der Junge befremdet mit scheu niedergeschlagenen Augen in einer Ecke stehenblieb, bis niedergeschlagenen Augen in einer Ecke stehen blieb, bis man ihn zu einem der Schachtische hinrief."5

„hinrief“ ist ein Kompositum aus dem Adverb hin und dem Verb rufen und meint im transitiven Sinn, dass man Mirko von seinem Standort herbeizitierte. Die Herkunft stammt aus der oberdeutschen lautmalenden Schreibsprache, wo ,(h)ruofan“, vor allem in Österreich und Bayern, zu den noch heute gebräuchlichen regionalen sprachlichen Eigenheiten zählt.

$\mathrm{Zu}$ diesen sprachlichen Eigenheiten zählt bei Stefan Zweig auch der unterschiedliche Gebrauch der Interfixe be- und ge-. Zum Beispiel heißt es: „Es dauerte eine Weile, ehe man dem Unbelehrten begreiflich machen konnte, $[\ldots]^{\text {]66 }}$

Es handelt sich bei dem Begriff des Unbelehrten um ein Kompositum, bestehend aus dem Präfix un-, das eine Negation kennzeichnet, und dem Interfix be-, das als Fugenelement zwischen substantivierten Adjektiv lehrten steht.

Einen ähnlichen linguistischen Sonderfall bietet der Begriff fachmäßig:

„[...] den jungen Menschen in Wien von einem ihm bekannten ausgezeichneten kleinen Meister fachmäßig in der Schachkunst ausbilden zu lassen." ${ }^{\text {"7 }}$

Das selten gebrauchte Adjektiv „fachmäßig“ ist ebenfalls typisch für die Oberdeutsche Sprache und wird im Sinne von fachmännisch benutzt.

Der zweite Teilbereich der von Stefan Zweig verinnerlichten deutschen Sprache steht in der Tradition der Meißnischen Kanzleisprache,${ }^{8}$ die auch die Meißnische sächsische Kanzleisprache genannt wird. ${ }^{9}$ 
Wenn man bei dem o. g. Beispiel „fachmäßig“ bleibt, dann wird der Dualismus zwischen der Oberdeutschen Sprache und der sächsischen Meißnischen Sprache deutlich, wenn man in der Schachnovelle den folgenden Satz ließt: „Denn Czentovic brachte es nie dazu, auch nur eine einzige Schachpartie auswendig - oder wie man fachgemäß sagt: blind zu spielen." ${ }^{10}$

Hier wechselte Stefan Zweig von „fachmäßig“ aus der Oberdeutschen Sprache zu „fachgemäß“ der sächsischen Meißnischen Sprache und zeigte damit, dass er seine sprachlichen Wurzeln sowohl in der einen wie in der anderen Sprachausformung hatte. Die Kombination der beiden deutschen Sprachen erfolgte in der späteren Phase im oberdeutschen Sprachraum und ist als die Zeit der Katholische Aufklärung bekannt geworden. ${ }^{11}$

Die Katholische Aufklärung wurde in Österreich deshalb bedeutend, weil die Jurisprudenz, die Medizin und die Naturwissenschaften nur noch faktische Erkenntnisse verbreiteten, ohne die biblischen Relevanzkriterien $\mathrm{zu}$ verifizieren. Also mussten Neologismen generiert werden, um die realistischen Fakten sprachlich und schriftlich neu zu definieren.

Diese Entwicklung bewirkte, dass sowohl die Spätphase der oberdeutschen Literatursprache wie auch die sächsische Meißnische Sprache für das Werk von Stefan Zweig ausschlaggebend wurden und er schon als Kind die übergreifende Literatursprache des oberdeutschen Sprachraum verinnerlichte. ${ }^{12}$

\section{Historisierende Neologismen}

Am Beispiel der Clause von den „gerissensten Journalisten“ findet man in der Schachnovelle die Haltung des Historismus wieder: ,[...] aus dem auch nur ein einziges publizistisch brauchbares Wort herauszulocken selbst den gerissensten Journalisten nie gelang. “13

Die Herkunft von reißen und den im Text genannten Superlativ gerissensten stammt ursprünglich von dem Vorgang, Runen-Zeichen in Buchen-Stäbchen zu ritzen.

Zweig nimmt hier redensartlichen Bezug auf das sog. Germanentum, dass es aber in der historischen Wirklichkeit nie 
einheitlich gegeben hat. Eine Erklärung für diese Bezugnahme findet der Leser in Victor Klemperers LTI. Dort heißt es:

„Dagegen kann man sich Datierung „im Lenzig“ nur im Zusammenhang mit Bayreuth, der Wagnerstadt, leisten - die altdeutschem Monatsnahmen setzen sich allem Runengebrauch und SiegHeil-Geschrei zum Trotz in der allgemeinen Sprache nicht durch. ${ }^{14}$

Daher konnte Stefan Zweig historisierende Neologismen in seiner Novelle weiterhin verwenden, ohne befürchten $\mathrm{zu}$ müssen, in ein approximatives „Hiterdeutsch“ zu verfallen.

Eine weitere Variation eines historisierenden Neologismus ist der Rückgriff auf den Beruf des Barbiers.

„Nachdem er Mirko bei dem Dorfbarbier die struppigen strohblonden Haare hatte schneiden lassen, um ihn einigermaßen präsentabel zu machen, nahm er ihn in seinem Schlitten mit in die kleine Nachbarstadt,[...]“15

\section{Der Antisemitismus und die Folgen}

Stefan Zweig entdeckte als junger Erwachsener, dass er jüdischer Abstammung war und er bezeichnete sich selbst als „Jude aus Zufall“. Diese Selbsterkenntnis hatte für sein weiteres Lebenswerk u. a. auch sprachliche Konsequenzen. ${ }^{16}$

Bei der Analyse der sprachlichen Konsequenzen und dem daraus folgenden kreativen Sprachgebrauch von Stefan Zweig kann die Lektüre von Victor Klemperer LTI: Notizbuch eines Philologen entscheidende Hinweise geben. Denn Klemperer (1881-1960) und Stefan Zweig verband nicht nur das gleiche Geburtsjahr 1881, sondern auch ihre jüdische Abstammung.

Klemperer hat seine Notizen aus der NS-Zeit seinem Tagebuch anvertraut, und diese teils heiteren scharfsinnigen und spannenden Sprach- und Stilstudien liefern auch für die vorliegende Arbeit bedeutsame Hinweise. Denn Klemperer schilderte, wie die Propaganda des NS-Regimes mit euphemistischen Neologismen Politik machte. ${ }^{17}$

$\mathrm{Ob}$ jedoch Stefan Zweig kognitiv eine Abgrenzungsstrategie entwickelt hat, ist nur konkludent nachweisbar. Dass es jedoch solche 
Überlegungen bei intellektuellen jüdischen Menschen in Deutschland gegeben hat, ist bei Victor Klemperer nachzulesen. Denn einen solchen Abgrenzungsdisput gegen das „Hitlerdeutsch“ schildert Victor Klemperer in LTI im Kapitel Die Sprache der Sieger, als er mit „,der wirklich denkgeschult[en] und leidenschaftliche[n] interessierte[n] Germanistin Elsa Glauber" aneinander geriet. Doch voller Hochachtung bemerkte Klemperer, dass er sich nicht darüber klar war, ob Elsa Glauber „eine bessere Jüdin oder bessere deutsche Patriotin war" ${ }^{\text {“ }}{ }^{18}$

Elsa Glauber verstieg sich nämlich $\mathrm{zu}$ einem Gebrauch des Adjektivs fanatisch genau in der Art, wie die Nazis dieses Wort im „Hitlerdeutsch“ benutzten. Sie sagte: „Sie müssen denken lernen wie ich, sie müssen Goethe lesen wie die Bibel, sie müssen eine fanatische Deutsche sein! ${ }^{119}$

Diese Schilderung zeigt, wie die stetige und überall präsente Propaganda in die deutsche Sprache eindrang und fanatische Nationalsozialisten kaum mehr von fanatischen Deutschen $\mathrm{zu}$ unterscheiden waren. Den Grund für diese Schwierigkeit der Unterscheidung lieferte nach dem Krieg Hannah Arendt. ${ }^{20}$ Sie war 1961 für das Magazins „The New Yorker" beim Prozess gegen Adolf Eichmann (1906-1962) als Pressebeobachterin anwesend.

Hannah Arendt nahm zweimal an den Sitzungen des Gerichts teil. In ihrem 1963 erschienenen Buch ,Eichmann in Jerusalem “ bzw. in der englischen Version „A Report on the Banality of Evil" fasste sie ihre gewonnenen Eindrücke zusammen. ${ }^{21}$

Ihren persönlichen Eindruck vom Angeklagten, seinem Lebenslauf und seiner Tätigkeit als Organisator der Vertreibung von Juden, sowie als Koordinator sämtlicher Deportationstransporte in die verschiedenen KZ-Lager, fasste Hannah Arendt in einem Wort zusammen und nannte ihn einen „Verwaltungsmassenmörder“. Aber das Buch entwickelte sich $\mathrm{zu}$ einem Medienereignis mit Skandalqualität, als Hannah Arendt diesen als Kriegsverbrecher verurteilten Adolf Eichmann in ihrem Buch als einen ,normalen“ Menschen bezeichnete. Zwar habe Eichmann eine Karriere im SS- Apparat angestrebt, doch habe er dabei keine Mord-Motivation gehabt und sei auch nicht von einem übermäßigen 
Antisemitismus beherrscht gewesen. Eichmann sei also als psychisch normal zu bezeichnen und nicht als ein Ungeheuer oder gar ein Dämon. Er habe nur seine Pflicht aus seinem vertraglichen Arbeitsverhältnis und auf Grund von speziellen Weisungen erfüllt, dabei habe er nur den Befehlen und dem damals gültigen Gesetz gehorcht. ${ }^{22}$

Denn Adolf Hitler habe die Gesetze so verändert, dass Eichmann keine eigenen ethischen Vorstellungen aus sich selbst heraus habe entwickeln können. Aus seiner Position heraus habe er sich wegen der so geschaffenen gesetzlichen Strukturen nicht in der Lage gesehen, daran etwas zu ändern. Eichmann sei unfähig, selbst zu denken, dieses zeige sich vor allem an der Verwendung klischeehafter Phrasen und einem Verstecken hinter der Amtssprache. Diese Amtssprache sei allen Teilnehmern der Wannseekonferenz eigen gewesen und nicht nur Adolf Eichmann. So hätten die Spitzen von Ministerien, Justiz und der Wehrmacht die „Endlösung der Judenfrage“ beschlossen, ohne innere persönliche Anteilnahme. Doch was diese verwaltungstechnische Formulierung bedeutet habe, sei allen Beteiligten bewusst gewesen. Sie hätten mit dieser euphemistischen formulierten Amtssprache der Ermordung aller Juden in Europa zugestimmt. Deshalb habe sich auch Eichmann danach als Vollstrecker dieses Beschlusses gefühlt und jede persönliche Verantwortung für die Ausführung des Beschlusses abgelehnt.

Doch Stefan Zweig wollte sich auf jeden Fall von einem fanatischen Deutschen unterscheiden, und so fügte er der Erwähnung des Fanatismus seine eigene Interpretation hinzu, als er in der Schachnovelle das Phänomen einer sozial unverträglichen Verhaltensweise - den fanatischen Ehrgeiz - behandelte. Denn Zweig machte diese fanatische Verhaltensweisen an der Person McConnor fest und schrieb: „In diesem Augenblick wusste ich, dieser fanatisch Ehrgeizige würde [...] [23

Dabei steht jedoch das von Sigmund Freud definierte „Es“ im Fokus, denn aus Stefan Zweigs Vita ist die innige Bekanntschaft mit Sigmund Freud belegt. ${ }^{24}$ 


\section{Die nazistische Sprache und die Generierung von Neologismen}

Einer der Begründer des verheerenden antijudaistischen Gedankengutes war Georg von Schönerer (1842-1921). Dieser adlige österreichische Politiker war um die Jahrhundertwende der Führer der Deutschnationalen Bewegung und später der Alldeutschen Vereinigung. Schönerer bekämpfte den politischen Katholizismus und die Installierung eines „Christlichen Ständestaates“, aber genauso trat er als ein radikaler Antisemit hervor. Durch diese Haltung übte er einen starken Einfluss auf Adolf Hitler aus, der ihn als eines seiner Vorbilder ansah. ${ }^{25}$

Einen solchen Einfluss auf Adolf Hitler hatte auch Karl Lueger (1844-1910). Lueger war von 1897 bis 1910 Wiener Bürgermeister und benutzte als politische Wahlkampfstrategie den von Georg von Schönerer angefeuerten Antisemitismus. Dabei wurde die jüdische Minderheit mit mittelalterlichen Begriffen des Antijudaismus und Antisemitismus beleidigt, indem man sie u. a. mit Tiermetaphern wie „Judensau“ bezeichnete. Mit diesen Begriffen sollten die Juden verhöhnt, ausgegrenzt und gedemütigt werden, da das Schwein in der alten abrahamitischen Religion bis heute als unrein gilt und einem Nahrungstabu unterliegt. Nach Karl Luegers frühem Tod, wegen seiner Zuckerkrankheit, nahm eine riesige Menschenmenge, darunter auch Adolf Hitler, an seiner Beerdigung teil. ${ }^{26}$

Diese Art der Diktionen von Karl Lueger und Georg von Schönerer machte sich Hitler zu eigen, indem er in sein „Kampfbuch“, wie Klemperer es nannte, verunglimpfend hinein schrieb:

„Der Jude ist und bleibt der typische Parasit, ein Schmarotzer, der wie ein schädlicher Bazillus sich immer mehr ausbreitet, sowie nur ein günstiger Nährboden dazu einlädt.“'27

Dieses Zitat strotzt vor Ausdrücken der Biologie und imitiert ihre wissenschaftliche Fachsprache. Victor Klemperer fand für solche pseudowissenschaftlichen Sätze im Kapitel Die Wechselbrause seiner LTI einen originellen Neologismus. Er schrieb: „Ich nenne das den auf jede Disziplin der Wissenschaft, Philosophie und Kunst anwendbaren nazistischen Tiefenstil.“ ${ }^{28}$ 
Hitler hat mit diesem „nazistischen Tiefenstil“ seinen Hetzreden einen pseudowissenschaftlichen Anstrich gegeben, um seine wahren Intensionen euphemistisch zu verbrämen. Denn seine Aussagen sollten für die Zuhörer wichtig und richtig klingen und zudem wissenschaftlich begründet sein.

Also gab es eine notwendige Abgrenzung eines „Juden aus Zufall“, wie Stefan Zweig sich selbst bezeichnete, gegen den „,nazistischen Tiefenstil“ in Österreich. Doch dieser österreichische Prozess lag nicht im Fokus Victor Klemperers in der Zeit der deutschen Judenverfolgung. Deshalb kam er in seinem Buch LTI, im Kapitel Der Fluch des Superlativs, zu seiner nicht ganz zutreffenden Bewertung des von Stefan Zweig gepflegten Schreibstils. Er schrieb: ,[...] Leute, die viel auf exklusive Eleganz und Neuheit ihres Stils geben, so Stefan Zweig, so Rathenau, wenden ihn (d. h. den Superlativ) an. “29

Diese Äußerung kann als Vorwurf der Oberflächlichkeit verstanden werden, aber unter Berücksichtigung der politischen Gegebenheiten und des frühen Antijudaismus in Österreich wird aus „exklusive Eleganz und Neuheit“ ein Lob für einen gelungenen neologischen Schreibstil.

Um seine kriegerischen Usurpationsabsichten $\mathrm{zu}$ verschleiern, verwendete Hitler gern euphemistisch den Begriff „Anschluss“. Der Begriff stammt ursprünglich aus der Elektrotechnik und signalisiert Modernität. Doch Hitler verwendete „Anschluss “ für die Usurpation seiner „Heimat Österreich“ und auch für die Usurpation von Tschechien.

Mit dieser Thematik beschäftigte sich auch Stefan Zweig in der Schachnovelle, allerdings deskriptiv, als er Dr. B. berichten lässt:

„Sie mussten aber erfahren haben, dass wir die Strohmänner, die Verwalter und Vertrauten ihrer erbittertsten Gegner gewesen, und was sie von mir zu erpressen hofften, war belastendes Material: Material gegen die Klöster, denen sie Vermögensschiebungen nachweisen wollten, Material gegen die kaiserliche Familie und all jene, die in Österreich sich aufopfernd für die Monarchie eingesetzt. “30

Denn das NS-Regime war ständig von einem Bankrott bedroht, und so war es eine der ersten schändlichen Handlungen der Nazis nach 
dem „Anschluss“, die Finanzmittel der erklärten Gegner, beispielsweise der Kirchen und der Monarchie, gewaltsam zu akquirieren. ${ }^{31}$

Ebenso stammt der Begriff der „Gleichschaltung“ aus der Elektrotechnik und unterstreicht vordergründig eine Technikversessenheit. Diese „Technikversessenheit“ war jedoch eine Neologismus aus dem NS-Amtsdeutsch.

Victor Klemperer setzte sich mit der technischen - und mechanisierten Sprache des Hitlerdeutsch in LTI auseinander, indem er das schwache Verb „gleichschalten “ untersuchte. Er schrieb:

„Das eindeutige Mechanisieren und der Person selber bleibt der LTI vorbehalten. Ihre charakteristischste, wahrscheinlich auch früheste Schöpfung auf diesem Felde heißt ,gleichschalten“. Man sieht und hört den Druckknopf, der Menschen, nicht Institutionen, nicht unpersönliche Behörden, in gleichförmige automatische Haltung und Bewegung versetzt: Lehrer verschiedener Anstalten, Gruppen verschiedener Angestellter des Justiz-, des Steuerdienstes, Mitglieder des Stahlhelms, und der SA usw. usw. werden beinahe in infinitum gleichgeschaltet. ${ }^{\text {" } 32}$

Tatsächlich bedeutete also „Gleichschaltung“ im Hitlerdeutsch „Ausschaltung“ von Mitgliedern anderer Parteien, Gewerkschaften und auch von Jugendverbänden. Bei geringstem Widerstand wurden Personen, wenn sie nicht sofort umgebracht wurden, ins Gefängnis oder in eines der Konzentrationslager gesteckt.

\section{Die Wurzel der nazistischen Sprache}

Victor Klemperer schrieb in LTI im Kapitel Die deutsche Wurzel über die Entstehung des Rassismus in Deutschland. ${ }^{33}$

Dabei formulierte er, dass die ,[...]deutsche(n) Grundeigenschaft der Maßlosigkeit, der Überkonsequenz, des Ins- Grenzenlose- Langenes gab den üppigsten Nährboden der Idee her.“34

Und Klemperer verfolgte den Beginn der narzisstischen Sprache in ,ihren theoretischen Ausdruck rückwärts, so geht die gerade Linie in ihren Hauptetappen von Rosenberg über den wahl- deutschen Engländer Houston Stewart Chamberlain auf den Franzosen Gobineau zurück. ${ }^{\text {(335 }}$ 
In der weiteren Folge wird Joseph Arthur de Gobineau als der verantwortliche Urheber der blutigen NS-Doktrin ausgemacht und Klemperer meinte: „Hier ist alles gegeben, was das Dritte Reich zu seiner philosophischen Begründung und für seine Politik braucht[...]“ Denn ,[...] alle spätere vor- nazistische Ausbauen und Anwendungen der Lehre geht immer wieder auf diesen einen Gobineau ${ }^{36}$ zurück, [... $]^{\text {“. } 37}$

Dabei nahm Klemperer Bezug auf den Germanisten Wilhelm Scherer (1841-1886), dessen Werk er in sein innerdeutsches Exil in das Dresdner Judenhaus gerettet hatte. Denn Scherer beschrieb die Maßlosigkeit der Deutschen: „Maßlosigkeit scheint der Fluch unserer geistigen Entwicklung. Wir fliegen hoch, und sinken um so tiefer" und weiter: „Wir gleichen jenem Germanen. der im Würfelspiel all sein Besitztum verloren hat und auf den letzten Wurf seine eigene Freiheit setzt und die auch verliert und sich willig als Sklave verkaufen lässt. So groß - fügt Tacitus, der es erzählt, hinzu: ,,[...] ist selbst in schlechtere Sache die germanische Hartnäckigkeit; sie selbst nennen es Treue. “38

Also liegt eine der Wurzeln der deutsche Wurzel in einem „entscheidenden Charakterzug der deutschen Geistesbewegung“ und der „,heißt Grenzenlosigkeit“". ${ }^{39}$

\section{Die zwiespältige deutsche Romantik}

Auf Wilhelm Scherers Zitat aufbauend, der es wiederum bei Tacitus gefunden hatte, fuhr Victor Klemperer fort und schrieb, dass scheinbar „die Maßlosigkeit der Fluch unserer geistigen, in Wellenform verlaufenden, Entwicklung zu sein“ scheine und fuhr fort: „Die furchtbare Anklage besteht zu Recht, trotz aller von der Romantik geschaffenen Werte. “40

Ergo fand Klemperer: ,[...] alles, was den Nazismus ausmacht, ist ja in der Romantik keimhaft enthalten: die Entthronung der Vernunft, die Animalisierung des Menschen, die Verherrlichung des Machtgedankens, des Raubtiers, der blonden Bestie [...] $]^{\text {‘4 } 1}$

Damit ging Victor Klemperer einer weiteren Wurzel des deutschen Antisemitismus nach und widmete dem Roman von Max René Hesse Partenau in LTI ein eigenes Kapitel. Dabei bezeichnete er das 
Werk Partenau als „Vorwegnahme der Sprache, der Gesinnungen des Dritten Reichs“. ${ }^{42}$

Klemperer zitiert Friedrich Martin von Bodenstedt ${ }^{43}$ (1819-1892): „Aus der selben Ackerkrume wächst das Unkraut wie die Blume. Und die deutsche Wurzel heißt Romantik." Damit meinte Klemperer nicht die Epoche der Romantik, die für das romantische Denken und für die romantische Poesie steht. Zweig und Klemperer erlebten in „GroßDeutschland“, dass auf „der selben Ackerkrume“ wie das Unkraut der Nazis auch „die Blume“ wuchs. Deshalb schrieb Victor Klemperer, nicht nur an dieser Stelle - er leitet seine Feststellung etliche Male mit den gleichen Ergebnissen ab - dass die romantischen Vorstellungen des deutschen Volkes eine der Ursachen der „Hitlerei“ waren. ${ }^{44}$

Dieses war die Ausgangssituation der Sprachentwicklung, die auf Stefan Zweig und auch auf die anderen deutschsprachigen Schriftsteller einwirkte. Zweig, der in Salzburg wohnte, nur wenige Kilometer von Hitlers Wohnsitz auf dem Obersalzberg entfernt, nahm die nationalsozialistische Bedrohung sehr ernst und sah darin ein „Vorspiel [zu] viel weiterreichenden Eingriffen“. ${ }^{45}$

Stefan Zweig zeigte sich von den „weiterreichenden Eingriffen“ tief erschüttert und verließ Österreich, um zunächst nach London zu emigrieren. „Am Tage, da ich meinen Pass verlor, entdeckte ich mit achtundfünfzig Jahren, dass man mit seiner Heimat mehr verliert als einen Fleck umgrenzter Erde. “46

Denn Stefan Zweig war nun nicht nur räumlich, sonder auch formal von seinen lebendigen deutschsprachigen Wurzeln getrennt und stand nun vor der Aufgabe, seine Anliegen in seinen Büchern so klar zu formulieren, dass seine Aussagen unmissverständlich authentisch erschienen. Dabei enthielt sich Zweig jedweder romantischer Ideen und aller Begriffe des ,nazistischen Tiefenstils“.

\section{Philosophie vs. Weltanschauung}

Der Augen- und Zeitzeuge Victor Klemperer schrieb in seinem Buch LTI über die Philosophie, dass im NS-Regime die Philosophie durch den Begriff „Weltanschauung“ ersetzt wurde: „Philosophie dagegen wird totgeschwiegen, wird durchgängig ersetzt durch „Weltanschauung.“47 
Klemperer zitierte als Beispiel für diese Entwicklung den NSIdeologen und späteren Außenminister Alfred Rosenberg. ${ }^{48}$

„Die Berufsphilosophen, lehrt Rosenberg, begehen durchweg einen doppelten Fehler. Erstens begeben sie sich auf die „Jagt nach der sogenannten einen, ewigen Wahrheit“. Und zweitens fahnden sie ,auf rein logischem Wege, indem sie von Axiomen des Verstandes weiter und weiter schließen. “49

Rosenberg meine damit, dass nicht die Logik und der Verstand Gegenstand der Philosophie sein sollten, sondern die unbeweisbaren Thesen, die als leicht einsichtige Wahrheiten verstanden und als allgemein gültig und richtig erkannt werden sollten. Deshalb brauche die Richtigkeit der nazistischen Weltanschauung nicht bewiesen werden. Rosenbergs Simplifizierung ist nichts anderes als eine euphemistische Umschreibung für die angebliche Meinung des ungebildeten Volkes. Denn damit lag die Deutungshoheit bei dem sog. „Führer“ oder der veröffentlichten Propagandameinungsbildung. Im Bereich der Literatur sollten ganz im Sinne einer nationalsozialistischen Weltanschauung missliebige Werke als „entartete Kunst“" gebrandmarkt werden. So wurden auch Stefan Zweigs Werke im wörtlichen Sinn gebrandmarkt, als seine Bücher bei der Salzburger Bücherverbrennung in der Altstadt verbrannt wurden. ${ }^{50}$

Victor Klemperer machte für den Wortschatz der Nationalsozialisten die Unausweichlichkeit einer willentlich getroffenen Entscheidung und deren unüberbrückbare Opposition $\mathrm{zu}$ anderen Weltanschauungen verantwortlich. Einer Ideologie kann man anhängen, aber Weltanschauungen werden geteilt - oder auch nicht, und deshalb bezeichneten die Nazis ihre Weltanschauung selbst nicht als eine Ideologie.

In LTI machte Victor Klemperer darauf aufmerksam, dass alle möglichen Positionen und selbst die Philosophie zu bloß konkurrierenden Anschauungen degradiert wurden, gegen welche sich der Nationalsozialismus als „totale Weltanschauung“ durchsetzen sollte. Und zwar sollte dies durch Überzeugung und Propaganda der Zaudernden gelingen. Aber all jenen, die die Weltanschauung des Nationalsozialismus 
nicht teilen wollten oder sollten - wie z. B. die Juden -, drohte die physische Vernichtung.

Stefan Zweig setzte dagegen bei seinen Lesern ein Verständnis bzw. die Möglichkeit einer Einordnung der Gedanken in einen übergeordneten Zusammenhang voraus. $\mathrm{Er}$ erinnerte an die philosophischen Schulen der antiken Welt, indem er schrieb:

„[...] außerdem erledigte er (gemeint ist Czentovic) immer so eilig und stoßhaft seine peripatetische Deckrunde, dass man ihm hätte im Trab nachlaufen müssen, um ihn ansprechen zu können. “51

Mit dem Adjektiv „peripatetisch“ wird nicht nur auf die Schule des Aristoteles und auf dessen Lehre Bezug genommen, sondern Stefan Zweig bezog sich auf die Annahme des peripatetischen Gehens. Die Romanfigur des Czentovic soll also einerseits in seine Gedanken versunken über das Deck gegangen sein, wie der Philosoph Aristoteles, andererseits aber „eilig und stoßhaft“, wie man sich das Marschieren der deutschen Soldaten vorstellt. Zweig bediente sich hierbei der populären Etymologie, die den Namen Peripatos direkt von peripatein $=$ umher wandeln ableitet. Heute leitet man wie im Buch Aristoteles - Peripatos, stattdessen das Substantiv Spaziergang davon ab. ${ }^{52}$

Also bemerkt ein Rezipient den Gegensatz zwischen „stoßhaft“ gehen, als ob ein Soldat im Stechschritt marschieren würde, und der „peripatetische(n) Deckrunde“, die eher auf einen Spaziergang wie in einer Wandelhalle hinweist. ${ }^{53}$

Für den Rezipienten bestünde dieser Gegensatz nicht, hätte Zweig noch das Adverb eigentlich hinzugefügt. Dann hätte Czentovic „eilig und stoßhaft" die Deckrunde erledigt, wo doch eigentlich ein ,peripatetisches Wandeln " vorgesehen war.

In diese Reihe von philosophischen Kenntnissen fällt auch die Kenntnis über die Lehren der Physiognomie des aus Deutschland geflüchteten und in Paris praktizierenden deutschen Arztes und Anatomen Gall. Denn Stefan Zweig schrieb:

„In früheren Zeiten physiognomischer Leidenschaft hätte ein Gall vielleicht die Gehirne solcher Schachmeister seziert, um festzustellen, ob bei solchen Schachgenies eine besondere Windung in der grauen Masse 
des Gehirns, eine Art Schachmuskel oder Schachhöcker sich intensiver eingezeichnet fände als in anderen Schädeln. ${ }^{\text {"55 }}$

In der ersten Clause zog Zweig die ,physiognomische[r] Leidenschaft" an, allerdings mit der Einschränkung, dass er sich auf die „früheren Zeiten“ beziehe. Eine zeitliche Orientierung gibt der Hinweis auf ,ein Gall“. 55

Es ist Stefan Zweig spekulativ zuzutrauen, dass er mit dieser Clause seine gebildeten Leser-Schichten, an den - schon in der Zeit der Weimarer Republik - verdrehten Gebrauch des Begriffs Physiognomie erinnern wollte. Denn damals gab es einen prekären Übergang von der Physiognomik hin zu einer rassistischen antisemitischen Lehrmeinung. ${ }^{56}$

\section{Psychiatrische Neologismen}

Stefan Zweig diagnostizierte seine fiktiven Figuren, wie sein Freund und Wiener Mitbürger Sigmund Freud (1856-1939). Denn Freud entwickelte Methoden, die damals für die Behandlung von psychischen Störungen geeignet schienen. Nach diesem Vorbild diagnostizierte Stefan Zweig in der Schachnovelle die äußeren Veränderungen McConnors und dessen dunkle Charakterseiten:

„In diesem Augenblick wusste ich, dieser fanatisch Ehrgeizige würde, und sollte es ihn sein ganzes Vermögen kosten, gegen Czentovic so lange spielen und spielen und spielen, einfach oder doubliert, bis er wenigstens ein einziges Mal eine Partie gewonnen. “57

Die Clause ,[...] dieser fanatisch Ehrgeizige würde[...]“ erscheint als eine mögliche ironisierende Einlassung in Richtung des Fanatismus des NS-Regimes, aber Stefan Zweig erklärt den Grund von McConnors Fanatismus nicht.

Der Fanatismus aber lag im NS-Regime tagtäglich in der Luft. Ob in einzelnen Reden, oder auf Flugblättern, ob in Begriffen und Wörtern, stets sollte der „fanatische Wille“ spürbar werden.

Denn der Fanatismus zeigte sich bei den Rednern, z. B. bei Hitler, seinen scheinbar zornigen Wutanfällen, die oft den Eindruck von Ekstasen hinterließen. Es schien, als nähmen sie ihre Visionen für Realität und artikulierten ihre Einbildungen als Prophezeiungen. Sie waren Enthusiasten, die ihren Irrsinn mit Mord durchsetzten. Das führte zu 
ständigen Suggestionen, und Victor Klemperer schrieb in seinem Werk LTI: „Niemals vor dem Dritten Reich wäre es jemanden eingefallen, fanatisch als ein positives Wertwort zu gebrauchen. ${ }^{\text {"58 }}$

Denn der positive ehrgeizige Lebenstrieb (des Eros) begründete einerseits McConnors beruflichen Erfolg. Andererseits, der negativen Trieb (des Thanatos) die Form seiner Maßlosigkeit. Denn der gesamten Besitz McConnors ist vom „fanatischen Ehrgeiz“ bedroht, der ihn zum Sklaven seines zwanghaftes Spielen werden lässt. Sein pathologisches Spielen ist durch seine Unfähigkeit gekennzeichnet, dem Impuls zum Spiel zu widerstehen.

\section{Linguistische Neologismen}

Im Wörterbuch der Abkürzungen bezeichnet der linguistische Begriff „Abbreviatur“ eine Abkürzung, allerdings nur in der schriftsprachlichen Abkürzungssprache.

Die NS-Propaganda brachte es im Erfinden von Abbreviaturen zu einer zweifelhaften Meisterschaft. Victor Klemperer schrieb in seinem Werk LTI: „Kein vorhergehender Sprachstil macht einen so exorbitanten Gebrauch von dieser Form wie das Hitlerdeutsch." ${ }^{\text {(59 }}$

Als Beispiele für dieses „Hitlerdeutsch“ zieht Klemperer die folgenden Abbreviaturen heran: „Kradschütze oder Mannschaft am MG, Glied der HJ oder der DAF - man ist immer ,verschworene Gemeinschaft. “60

Doch Klemperer berichtete auch von einem internen Widerstand von NS-Kadern gegen diese Praxis, denn in einem Zeitungsartikel der „DAZ“ (Deutsche Allgemeine Zeitung) hieß es:

„Später im März 1944, kam es einmal zu einer ernsthaften öffentlichen und offiziellen Warnung vor dem missbräuchlichen Übermaß der „Stummelwörter“, wie die Abbreviaturen hierbei genannt wurden. “61

In der weiteren Folge wurden Abbreviaturen als „sprachverhunzende Kurzwörter“ bezeichnet und als Beispiel „Hestra der Wigru" angeführt. ${ }^{62}$

In offiziellen Texten wurde jedoch der deutschsprachige Begriff „Kurzwort“ benutzt. Es kann also festgehalten werden, dass die Nazis den 
Begriff „Abbreviatur“ wegen seiner lateinischen Etymologie nicht verwendeten.

So verwendete Stefan Zweig in der Schachnovelle den Begriff „Abbreviatur" für das nicht vollständige oder nur verkürzte Wissen von Czentovic über die Raffinessen des Schachspiels: „[...] eine merkwürdige und durchaus einmalige Abbreviatur der Welt. “63

Zweig meinte damit, dass das „sprachverhunzende Hitlerdeutsch“ - ,,...] eine merkwürdige und durchaus einmalige Abbreviatur der Welt"“ sei.

\section{Neologismen aus dem Französischen}

Stefan Zweig beherrschte die französische Sprache perfekt in Wort und Schrift. Er schrieb 1904 beispielsweise seine Dissertation über Die Philosophie des Hippolyte Taine und übersetzte anschließend die Werke Verlaines, Baudelaires und Émile Verhaerens.

Mit dem französisch stämmigen, gehobenen Adjektiv „prononcieren“ sprach Stefan Zweig die deutlich gerötete Gesichtsfarbe seiner Figur McConnor an:

„Er hieß McConnor und war ein schottischer Tiefbauingenieur, der, wie ich hörte, bei Ölbohrungen in Kalifornien sich ein großes Vermögen gemacht hatte, von äußerem Ansehen ein stämmiger Mensch mit starken, fast quadratisch harten Kinnbacken, kräftigen Zähnen und einer satten Gesichtsfarbe, deren prononcierte Rötlichkeit wahrscheinlich, zumindest teilweise, reichlichem Genuss von Whisky zu verdanken war." 64

Der französische Begriff prononcer bedeutet übertragen "aussprechen“ und ist aus der lateinischen Vokabel pronuntiare, die ebenfalls ,aussprechen“ bedeutet und auch als „bekannt machen“ übersetzt wird. Demnach lautet dieser Satz: ,[...] und einer satten Gesichtsfarbe, deren ausgesprochene Rötlichkeit wahrscheinlich [...]“. Auf den ersten Blick ist die französische Begriffsvariante eine eher ungewöhnliche Beschreibung von McConnors Hautfarbe, und dieser Ausdruck entspricht Zweigs bildungsbürgerlicher Einstellung durchaus.

Die schärfste Zuspitzung und Radikalisierung erfuhr, laut Christian Geulens Geschichte des Rassismus, das rassenbiologische 
Denken im Nationalsozialismus. Adolf Hitlers Buch Mein Kampf enthält ein Kapitel über die Eugenik, einschließlich der Konzentrationslager. Christian Geulen vermerkte in seinem Buch, dass Hitler die Hautfarben fälschlicherweise - als sozial-darwinistischen Überlebenskampf der menschlichen Rassen interpretierte. ${ }^{65}$

Eine andere frankophile Formulierung gibt zunächst Rätsel auf, da sie von dem heutigen Gebrauch der Umgangssprache stark abweicht. Stefan Zweig schrieb:

„Aber der Weltmeister ließ - ich hatte nach den Erzählungen meines Freundes derlei schon geahnt - gute zehn Minuten auf sich warten, wodurch allerdings sein Erscheinen dann erhöhten Aplomb ${ }^{66}$ erhielt." ${ }^{67}$

Mit dem heute veralteten Begriff Aplomb wird eine gewisse Unverfrorenheit oder Dreistigkeit sowie Frechheit beschrieben. Doch Stefan Zweig wollte bestimmt nicht die sichere Haltung und das Auftreten des aus Südslawien stammenden Beute-Germanen Czentovic und des nun reichsdeutschen Schachweltmeister Ausdruck verleihen und versah ihn mit einem geringschätzigen „erhöhten Aplomb“.

\section{Neologismen aus dem Alt-Griechischen}

Die Kenntnis der alt-griechischen Sprache und der griechischen Geschichte gehörte im 19. Jahrhundert zum Grundwissen eines akademisch gebildeten Bürgers. ${ }^{68}$

So wurden auch dem jungen Stefan Zweig im Gymnasium die Ideen des alt-griechischen Historikers Polybios (ca. 200 v. Chr.- ca. 120 v. Chr.) nahe gebracht. Polybios entwickelte ein Verfassungsschema für die griechischen Stadtstaaten, das zwischen verschiedenen Herrschaftsformen differenzierte und $u$. a. die Herrschaftsform der Tyrannei in der griechischen Antike beschrieb. Eine Reminiszenz findet man davon in der Schachnovelle, als der Erzähler in seinen Gedanken über das Schachspiel philosophiert und dabei alt-griechische Begriffe verwendet, die für ungebildete Leser unverständlich blieben. Zweig schrieb:

„Ich wusste wohl aus eigener Erfahrung um die geheimnisvolle Attraktion des ,königlichen Spiels“, dieses einzigen unter allen Spielen, 
die der Mensch ersonnen, das sich souverän jeder Tyrannis des Zufalls entzieht und seine Siegespsalmen einzig dem Geist oder vielmehr einer bestimmten Form geistiger Begabung zuteilt. “69

In der vierten Clause fällt der griechische Begriff der Tyrannis auf: ${ }^{70},[\ldots]$ das sich souverän jeder Tyrannis des Zufalls entzieht $[\ldots]^{6 / 71}$

Diese Formulierung mit dem griechischen Begriff der Tyrannis entspricht Stefan Zweigs konziliantem Konzept einer eher deskriptiven Kritik an den deutschen Verhältnissen. Doch er konnte sich auf seine Leserschaft verlassen, die es verstand, in der herrschenden Diktatur zwischen den Zeilen zu lesen.

\section{Neologismen aus dem Lateinischen}

Die lateinische Sprache hat in Europa viele Veränderungen durchgemacht. Zum Beispiel wurde der Satzbau vereinfacht, es wurden neue Wörter aus verschiedenen anderen Sprachen (z. B. aus dem AltGriechischen) aufgenommen und ihnen wurden neue Bedeutungen - also Neologismen - gegeben, die an die Stelle der alten Begriffe traten.

Der dem Naturalismus zuzurechnende Stefan Zweig nahm den melancholischen Dr. B. in die Schachnovelle auf, um der schachspielenden Gesellschaft auf dem Schiff ein naturalistisches Bild des nationalsozialistischen Grauens entgegen zu setzen. Ergo fand er eine Synthese zwischen Naturalismus und der Melancholie der in der Wiener Moderne verbreiteten Melancholie Durch die Verquickung der beiden Kunstrichtungen entstand eine neue dramatische Situation.

Die Schilderung der erst heute umfänglich bekannten Grausamkeiten des NS-Regimes wurden in der Schachnovelle noch nicht erwähnt, weil damals dafür noch keine gesicherten Erkenntnissen vorlagen. In diesen Sinne ist der folgende Satz zu verstehen, als Stefan Zweig-schrieb:

„Sie vermuten nun wahrscheinlich, dass ich Ihnen jetzt vom Konzentrationslager erzählen werde, in das doch alle jene übergeführt wurden, die unserem alten Österreich die Treue gehalten, von den Erniedrigungen, Martern, Torturen, die ich dort erlitten. ${ }^{\text {‘72 }}$

Neben der Marter werden im Text auch die Erniedrigungen genannt und auch die Torturen. Der Begriff Tortur ist im Latein genau so 
bekannt wie im Italienischen und meint das systematische Zufügen von Schmerzen, also Folter, die Leiden schafft. Die bildungssprachliche, gehobene Ausdruckweise kennt für den Vorgang der Marter auch den Begriff Drangsal.

Die Drangsale der Insassen der Konzentrationslager ausführlicher zu beschreiben, entsprach augenfällig nicht Stefan Zweigs Duktus. Denn in dieser Zeit gab es zwar Gerüchte über „Erniedrigungen, Martern und Torturen", aber keine sicheren Quellen. Als einzige Alternative zu den Gerüchten über die Drangsale kamen für Stefan Zweig nur seine Kenntnisse über die sogenannte Dreyfus-Affäre in Betracht. Denn der verbannte französische Offizier Alfred Dreyfus litt fast fünf Jahre (1894 bis 1899) lang auf der Teufelsinsel vor der Küste von FranzösischGuayana. Er war zu Unrecht wegen Landesverrat verurteilt worden und konnte nach der Isolationshaft kaum noch sprechen. Dies ist, auch nach heutigen Erkenntnissen, zurückzuführen auf den Mangel an Außenreizen wie z. B. an Kontakten $\mathrm{zu}$ anderen Menschen. Diese Art der Vereinsamung führt zu Halluzinationen und Denkstörungen, deshalb wird die Isolationshaft auch als weiße Folter bezeichnet. Dieses Vorgehen in Form der weißen Folter wird in der Schachnovelle angeführt, denn die Verhöre von Dr. B. zielten auf die Zerstörung seines psychischen Gleichgewichts ab, um ihn zu einem Geständnis und zur Zusammenarbeit mit den NS-Schergen zu bewegen. Nach der Vorlage des bedauerten Schicksals von Alfred Dreyfus wie es, in der veröffentlichen Anklage von Emil Zola „J'accuse ...!" geschildert wurde, wird in der Schachnovelle die „Gehirnwäsche“ von Dr. B. beschrieben. Aber ein solches Vorgehen wie diese Monate dauernde Isolationshaft des Dr. B. kann für das NSRegime, wenigstens bis heute, nicht belegt werden. Denn um rasche Geständnisse zu erzielen, setzten die Nazis z. B. in Auschwitz die sogenannte Boger-Schaukel ein. ${ }^{73}$

Hermann Langbein schrieb in seiner Dokumentation Menschen in Auschwitz, dass die Boger-Schaukel aus zwei senkrechten Pfosten bestand, die in den Boden gerammt wurden, mit einer dazwischen befestigten Querstange. An diese Stange wurde der Häftling mit den Kniekehlen kopfüber aufgehängt, wobei seine Handgelenke an seine Fußgelenke oder an die Querstange gefesselt wurden. Boger selbst nannte 
das Folterinstrument „Sprechmaschine“. Der gefesselte Häftling wurde mit dem Ochsenziemer blutig geschlagen, um ,sein Geständnis" zu erlangen. Der Häftling erhielt ca. 25 Hiebe auf das nackte Gesäß, wobei durch die Wucht der Schläge dem Häftling oft das Rückgrat gebrochen wurde. Meist wurden auch die Nieren offen gelegt, aber als geringste Auswirkung wurden dem Häftling dabei die Hoden zerschlagen. Deshalb starb der Häftling danach langsam unter stundenlangen Qualen in der Baracke oder wurde gleich, weil er tot war, auf dem Appellplatz zur „Abschreckung“" aller liegen gelassen. ${ }^{74}$

\section{Fazit}

Stefan Zweig konnte dank seiner kulturellen Prägung durch die oberdeutsche Schreibsprache und die Meißnische sächsische Kanzleisprache, sowie die Sprache der Katholischen Aufklärung kreative Wortbildungen erschaffen. Aus dem Sprach-Portfolio seiner sprachlichen Wurzeln konnte er die ihm angemessen erscheinende Ausdrucksweise auswählen. Das kam seinem österreichischen Idiom entgegen, so wie es die oberdeutsche Schreibsprache seit jeher erlaubte. Denn die deutsche Sprache ist dank ihrer vielen Quellen schon seit jeher flexibel, und sie ist es bis heute geblieben. Dadurch kann sie die ständig wandelnde Lebenswirklichkeit darstellen und abbilden.

Zweig schuf seine Neologismen, indem er bestehende Begriffe durch Ableitung, Komposition oder Umdeutung, neu gestaltete. Oder indem er aus den von ihm beherrschten Fremdsprachen Begriffe entlehnte, um damit politische, kulturelle, aber auch wissenschaftliche Veränderungen neu zu beschreiben und zu bewerten.

Stefan Zweig hat als „gerissener" Gegner des NS-Regimes historisierende Neologismen in seiner Novelle verwendet, um ein germanisiertes Hitlerdeutsch $\mathrm{zu}$ vermeiden und keine approximative Deutung zuzulassen. Doch um eine kulturelle, aber auch wissenschaftliche Verortung $\mathrm{zu}$ beschreiben, benutzte er einen historisierenden Neologismus. Denn ein Pfarrer ging normalerweise zu einem Frisör, aber um den besonders kleinen Ort in Südslawien besser zu verorten und charakterisieren zu können, schickte Zweig seinen Protagonisten zum Barbier. 
Stefan Zweig entwickelte eine sprachliche Strategie gegen die Sprache, die Victor Klemperer „Hitlerdeutsch“ nannte. Ob jedoch Stefan Zweig kognitiv eine Abgrenzungsstrategie entwickelt hat, kann aus seinem Nachlass bisher nicht entnommen werden, es ist nur konkludent nachweisbar. Denn wie notwendig eine solche sprachliche Abwehr war, wurde den Menschen in Deutschland erst langsam bewusst, als sie kaum noch zwischen fanatischen Nationalsozialisten und fanatischen Deutschen unterscheiden konnten. An dem Beispielwort fanatisch ist ersichtlich, wie nahe die psychiatrische Wissenschaftssprache eines Sigmund Freud und die Literatursprache Stefan Zweigs, aber auch die nazistische Amtssprache beieinander lagen. Dies war nicht alleine der allseits präsenten Propaganda von Goebbels geschuldet, welche die deutsche Sprache nachhaltig schädigte, es war auch die NS-Amtssprache, die in die Umgangssprache der Menschen eindrang. Es war die „Banalität des Bösen", wie sie Hannah Arendt schilderte, da die Nazis den Völkermord mit ihrer euphemistischen Amtssprache $\mathrm{zu}$ einer „Endlösung“ und zu einem fabrikmäßig geplanten Akt werden ließen. Auch Stefan Zweig stemmte sich mit seinen Möglichkeiten, gegen diesen Sprachverfall, der auch in Österreich langsam in die deutsche Umgangssprache einsickerte. Indem er an der Kathexis der Romanfigur McConnor festmachte, wie seine Leser der Hybris der fanatischen Nationalsozialisten begegnen konnten, um deren rassistische Sprache zu desavouieren. Aber diese rassistische Sprache wurde nicht im NS-Regime entwickelt, sondern wurde von den Vorbildern des jungen Hitlers.

Denn Hitler wurde indirekt von Georg von Schönerer und direkt von Karl Lueger, dem Wiener Bürgermeister, stark emotional beeinflusst und machte deshalb deren Ideen zu seinen eigenen. Diese Ideen setzte er später um, nachdem er alle freiheitlichen Institutionen ,,gleichgeschaltet “ d. h. ,,ausgeschaltet “ hatte. Deshalb fehlen bei Stefan Zweig Sätze, in denen diese Wörter vorkommen. Weder der Erzähler in der Schachnovelle noch Dr. B. suchten "Anschluss“. Und bei der Synchronisation der Schachspielergruppe kam es zwar zu Verabredungen, aber nicht zu einer ,"Gleichschaltung “.

Die nazistische Ausdrucksweise war eine Vermischung der verwaltungstechnischen deutschen Amtssprache mit euphemistischen 
wissenschaftlichen Bezügen. Klemperer nannte diese Sprache den „,nazistischen Tiefenstil“. Damit zwangen die Nazis den Menschen ihren Willen auf, den sie fadenscheinig wissenschaftlich begründeten. Damit predigten sie z. B. die irrige These von einem Sozial-Darwinismus, der wissenschaftlich gar nicht existiert. Leider waren die oft nur minimal gebildeten Menschen nicht in der Lage, die Hetzreden richtig zu bewerten, und deshalb nahmen sie eine ,gleichförmige automatische Haltung und Bewegung“" (laut Klemperer) ein.

Die nazistische Sprache hat auch eine zweite, eher intellektuelle Wurzel. Denn der Rassismus kam über den französischen Amtsadeligen Joseph Arthur de Gobineau - dem Begründer des rassistischen Denkens und den Ansichten des deutsch-englischen Autors Houston Chamberlain zu Arthur Rosenberg. Der Chefideologe der NSDAP Rosenberg übernahm die Ideen seiner Vorgänger und setzte sie explizit auf die nazistischen Belange um.

Als Quintessenz benutzten de Gobineau, Chamberlain und Rosenberg die Maßlosigkeit im Denken und den hartnäckigen Willen, etwas Großes zu erreichen, und die damit verbundene Eigenschaft, sich dabei selbst stets treu bleiben zu wollen dazu, den Menschen eine neue Ideologie - die sie Weltanschauung nannten - einzuimpfen.

Die Romantik schließt die Kritik an der Vernunft ein, eine Haltung die weder bei Stefan Zweig noch bei Victor Klemperer zu finden ist. Ganz im Gegenteil: beide sind geprägt durch ihr Erleben in der NaziZeit und sind deshalb durch und durch Realisten. Es findet sich in ihren oben genannten \Büchern auch keine Betonung der Sinnlichkeit, wie es in der Romantik durchaus üblich war. Weder Zweig noch Klemperer traten für romantische Abenteuer ein oder für ein wunderbares phantastisches Erleben im Unbewussten. Vielmehr schilderten beide die schaurige Abwendung von der Zivilisation durch die Nationalsozialisten. Deshalb war Stefan Zweig der nationalsozialistische Missbrauch der deutschen Romantik, wie sie von Max René Hesse in Partenau beschrieben wird, auf das Äußerste suspekt. Die „Weltanschauung“ von Rosenberg, Gobineau und Chamberlain bedeutete auch für Stefan Zweig eine Perversion seiner deutschsprachigen Wurzel und die Entwertung der Epoche der Romantik, die hohe Wertvorstellungen geschaffenen hatte. 
Doch nun wuchs in Deutschland anstatt literarischer „Blumen“ das „Unkraut“ des „,nazistischen Tiefenstils“.

Stefan Zweig versuchte augenfällig, seine gebildete Leserschaft auf die epochalen gesellschaftlichen Fehlentwicklungen aufmerksam zu machen, die der Nationalsozialismus in Österreich und in der Welt anrichtete, indem man die tradierte Philosophie totschwieg und durch eine NS-Weltanschauung ersetzte. Diese NS-Weltanschauung wurde gelebt und entzog sich nach Rosenbergs Vorgabe jeder Kritik, indem sie die Wahrnehmung selbst bestimmte. Durch die Propaganda hatten die Nationalsozialisten die Möglichkeit, die Perspektiven in allen Lebensbereichen zu ihrem Vorteil umzuformen.

So setzte Zweig dem im Stechschritt marschierenden Schachweltmeister eine auf der Lehre des Aristoteles beruhende „peripatetische Deckrunde“ entgegen und erinnerte mit „ein Gall“ an den „nazistischen Tiefenstil“, der aus Biologie, Phänomenologie und Religion ein „gesundes Volksempfinden" machte. Damit meinte er die philosophischen Verdrehungen Rosenbergs und die missbräuchliche Umschreibung der nationalsozialistischen Weltanschauung auf der Basis der angeblich unverderbten Meinung des Volkes. Dieses ,gesunde Volksempfinden" hatte besonders in Rechtsfragen vor dem sog. Volksgerichtshof katastrophale Folgen für die dort beklagten Menschen.

Stefan Zweig zeigte mit dem „fanatischen Ehrgeiz“ McConnors dessen triebhaften Teil - des „Es“ - in seinem Charakter und dessen aggressive Impulse. Ob die Aggressionen McConnors angeboren waren, oder ob seine Unbeherrschtheit durch Frustrationen, wie bei den Offizieren in Max René Hesse Partenau, sich zu einem fanatischen Ehrgeiz gesteigert hatten, ließ Stefan Zweig dabei offen. Der Leser erkennt im Umkehrschluss, dass Zweig ein negatives Wertwort verwendete, um sich von der fanatisch ereifernden Veranlagung McConnors zu distanzieren. Zweigs neologische Wortwahl ,[...] dieser fanatisch Ehrgeizige...]“ basierte auf der Beschreibung des „Es“ aus der Psychoanalyse Sigmund Freuds. Denn die Figur des McConnor wird anhand seiner Kathexis geschildert, die sich in seiner Spiel- und Gewinnsucht äußert. 
Es scheint so, als fühlte sich Stefan Zweig geradezu eingeladen, bildungssprachliche Begriffe zu verwenden. Denn das „Hitlerdeutsch“ verwendete aus ideologischen Gründen keine Wörter, die etymologisch nicht von einem germanischen Wortstamm abzuleiten waren. Also verwendete Stefan Zweig den aus dem Latein abzuleitenden Begriff „Abbreviatur“ für das unvollständige oder nur verkürzt vorhandene Wissen der Raffinessen des Schachspiels bei seiner Figur des Czentovic.

Aber die Nationalsozialisten waren Meister der Abbreviatur, die eine wahre Abkürzungswut entwickelten, und damit die eigene Amtssprache verstümmelten und verhunzten. Deshalb darf man annehmen, Stefan Zweig sei der Meinung gewesen, dass das Hitlerdeutsch ,[...] eine merkwürdige und durchaus einmalige Abbreviatur der Welt" sei.

Die Ironie von Stefan Zweigs französischen Neologismen wird oft erst in der Verkürzung sichtbar. Zum Beispiel, wenn man den französischstämmigen Ausdruck der „prononcierten“ Hautfarbe McConnors als deskriptiven neologischen Ausdruck Zweigs gegen die Diskriminierung der verschiedenen Hautfarben begreift.

Denn die von Stefan Zweig gepflegte Sprache ist der kulturelle Gegenentwurf zur Rassenpolitik der Nazis. Dies macht beispielsweise der heute veraltete Begriff Aplomb deutlich. Damit wurde eine gewisse Unverfrorenheit, Dreistigkeit oder Frechheit beschrieben. Doch die Ironie von Stefan Zweigs Ausdrucksweise wird sichtbar, wenn man die Unverfrorenheit und Dreistigkeit und die verlogenen Frechheiten beschreiben soll, mit der der sog. „Führer“ und die anderen Rassisten der NSDAP ihren „Aplomb“ initiierten.

Stefan Zweig verweilte mit seinen Gedanken bei dem griechischen Historiker Polybios und verwendete den Neologismus der Tyrannei als Synonym für die Diktatur Adolf Hitlers, unter der die Menschen in Europa litten.

Dass Stefan Zweig die gängige Praxis im NS-Regime verabscheute, ist durch seine Emigration hinreichend belegt. Deshalb ist Latein im Werk von Stefan Zweig eine erweiterte wichtige Ausdrucksmöglichkeit, da im NS-Regime keine lateinischen Begriffe 
verwendet wurden. Stefan Zweig schilderte die Erniedrigungen, Martern, Torturen, weiße Folter und die erfolgte „Gehirnwäsche“ des Dr. B. im Kontext einer grausamen Isolationshaft des Alfred Dreyfus, denn diese war für Stefan Zweig die einzige sichere Quelle. Zwar hörten die Menschen Gerüchte und Vermutungen in Groß-Deutschland, aber wissenschaftlich gesicherte Erkenntnisse, wie z. B. über die Boger Schaukel, gab es zunächst nicht. Trotzdem ist die emotionslose Genauigkeit der Schilderung über die weiße Folter ein Verdienst, für das der Erzähler Stefan Zweig hoch zu loben ist. Dabei bleibt Stefan Zweigs Gesellschaftskritik im Tonfall konziliant und ist dennoch eindringlich. Aber unter den heute bekannten Realitäten betrachtet, ist die zunächst realistisch erscheinende Schilderung des Dr. B. eine nicht adäquate Fiktion. 


\section{Literaturverzeichnis:}

Primärliteratur:

Zweig, Stefan: Schachnovelle, Fischer Taschenbuch Verlag, Frankfurt am Main, 1974

Klemperer, Victor: LTI-Notizbuch eines Philologen, Reclam, Philipp, jun. Verlag, Ditzingen, 2009

\section{Sekundärliteratur}

Arendt, Hannah: Eichmann in Jerusalem und die Folgen, Suhrkamp Verlag, Berlin, 2000

Anz, Thomas: Verwirrung der Gefühle. Stefan Zweig und Sigmund Freud. Verlag Literatur Wissenschaft de Nr. 11, Marburg, 2006

Bamberger, Richard (et al.): Österreich-Lexikon, Österreichischer Bundesverlag, Wien und München 1968

Donath, Matthias et al: Martin Luther, 69. Capitel der Tischreden, 1563. In: Sächsische Mythen: Elbe, August, Eierschecke, Verlagsgruppe: Seemann Henschel GmbH \& Co. KG, Edition Leipzig, 2011

Wissenschaftlicher Rat der Dudenredaktion, Annette Klosa u. a. (Hrsg.): Duden, Deutsches Universalwörterbuch. Dudenverlag, Mannheim/Leipzig/Wien/Zürich 2001

Flashar, Hellmut, (Hrsg.): Aristoteles - Peripatos, Schwabe Verlag, Basel 2004.

Geulen, Christian: Geschichte des Rassismus, C.H. Beck-Verlag, München 2007. 1928.

Hitler, Adolf: Mein Kampf, Franz Eher Nachfolger, München,

Langbein, Hermann: Menschen in Auschwitz, Europa Verlag Hamburg, 1999.

Laplanche, Jean und J. B. Pontalis: Das Vokabular der Psychoanalyse, Suhrkamp, Frankfurt/Main, 1972

Rathenau, Walther: Staat und Judentum. Eine Polemik. In: Walther Rathenau: Gesammelte Schriften. Band 1: Zur Kritik der Zeit. Mahnung und Warnung. Verlag S. Fischer, Berlin 1925

Scherer, Wilhelm: Geschichte der deutschen Literatur, Th. Knaur Nachf., Berlin, 1930

Schneider, Jost: Sozialgeschichte des Lesens: zur historischen Entwicklung und sozialen Differenzierung der literarischen Kommunikation in Deutschland. Walter de Gruyter, Berlin 2004 
Schramm, Macdonald: Gall, Franz Joseph. In: Allgemeine Deutsche Biographie \& Neue Deutsche Biographie. Gesamtregister. Band 8, Verlag Duncker \& Humblot, München und Leipzig 1878 Neudruck 1912

Wehrli, Fritz (Hrsg.): Die Schule des Aristoteles. Texte und Kommentare, 10 Hefte, Schwabe Verlag, Basel; 1967-1969) sowie dazu erschienen 2 Supplemente. Ebenda 1974

Zweig, Stefan: Die Welt von Gestern,- Erinnerungen eines Europäers, Anaconda Verlag, Köln

1 Max René Hesse (1877-1952) war ein deutscher Arzt und Autor von ca. zehn Entwicklungs- und Gesellschaftsromanen.

2 Thomas Anz (geboren 1948) ist ein deutscher Literaturwissenschaftler und war zuletzt Professor für Neuere deutsche Literatur an der Universität Marburg.

3 Anz, Thomas: Verwirrung der Gefühle. Stefan Zweig und Sigmund Freud. Verlag Literatur Wissenschaft Marburg Nr. 11, 2006

4 Zweig, Stefan: Schachnovelle, Fischer Taschenbuch Verlag, Frankfurt am Main, 1974, S. 17

5 a. a. O. S. 13.

6 a. a. O. S. 14.

7 Ebd. S. 14.

8 Die sächsische Kanzleisprache fand erst nach 1522 von Deutschland kommend in Österreich Eingang. Martin Luther gestaltete in dieser Sprache seine Bibelübersetzung, die dadurch zum allgemeinen Standarddeutsch wurde. Denn es war Luthers Intension, die regionalen Dialektsprachen zu überwinden, weil er von allen deutschsprechenden Menschen verstanden werden wollte.

9 Donath, Matthias und Thieme, André: Martin Luther, 69. Capitel der Tischreden, 1563, In: Sächsische Mythen: Elbe, August, Eierschecke, Verlagsgruppe: Seemann Henschel, Edition Leipzig, 2011

10 Zweig, Stefan: Schachnovelle, Fischer Taschenbuch Verlag, Frankfurt am Main, 1974, S. 15.

11 Man wollte der Bevölkerung einen besseren Zugang zur Bildung ermöglichen, aber dennoch stand das kreationistische Weltbild der Katholischen Kirche im Mittelpunkt der Bemühungen, mit dem Ziel, die Gegenreformation zu fördern.

12 Zweig, Stefan: Die Welt von Gestern,- Erinnerungen eines Europäers, im Kapitel: Die Welt in Sicherheit, Anaconda Verlag GmbH, Köln, 2013, S.13ff

13 Zweig, Stefan: Schachnovelle, Fischer Taschenbuch Verlag, Frankfurt am Main, 1974, Seite 17.

14 Klemperer, Victor: LTI - Lingua Tertii Imperii. Notizbuch eines Philologen. Reclam Verlag, Leipzig, 1998, S. 337 f

15 Zweig, Stefan: Schachnovelle, Fischer Taschenbuch Verlag, Frankfurt am Main, 1974, Seite 12.

16 Um einen solchen Moment nachvollziehen zu können, sind die Worte von Walter Rathenau überliefert, denn er schrieb über seine gleich gelagerten Erfahrungen in seiner Jugendzeit: 
„In den Jugendjahren eines jeden deutschen Juden gibt es einen schmerzlichen Augenblick, an den er sich zeitlebens erinnert: wenn ihm zum ersten Male voll bewußt wird, daß er als Bürger zweiter Klasse in die Welt getreten ist und keine Tüchtigkeit und kein Verdienst ihn aus dieser Lage befreien kann.“

Rathenau, Walther: Staat und Judentum. Eine Polemik. In: Walther Rathenau: Gesammelte Schriften. Band 1. Zur Kritik der Zeit. Mahnung und Warnung. Verlag S. Fischer Berlin 1925, S. 188F.

17 Klemperer, Victor, LTI: Notizbuch eines Philologen, Reclam, Philipp, jun. Verlag, Ditzingen, 2009 S. 337f.

18 Klemperer, Victor, LTI: Notizbuch eines Philologen, Reclam, Philipp, jun. Verlag, Ditzingen, 2009 S. 256.

$\mathrm{Ab}$ hier wird in dieser Arbeit nur noch „LTI“ zitiert, ohne den Untertitel: Notizbuch eines Philologen.

19 LTI, S. 257.

20 Hannah Arendt: (1906-1975) war eine deutsch-jüdische und amerikanische Wissenschaftlerin und Publizistin für Politische Theorie und Philosophie.

21 Vgl. Arendt, Hannah: Eichmann in Jerusalem und die Folgen, Suhrkamp Verlag, Berlin, 2000

22 LTI, S. 231.

23 Zweig, Stefan: Schachnovelle, Fischer Taschenbuch Verlag, Frankfurt am Main, 1974, S. 38.

24 Gemeint ist die 1923 erschienene Schrift Sigmund Freuds Das Ich und das Es. Dieses neue Strukturmodell der Psyche wird auch als das „Drei-Instanzen-Modell““ bezeichnet. Freud unterscheidet dabei die Triebe Eros und Thanatos. Eros der Lebenstrieb dient der Selbst- und der Arterhaltung, und der Todestrieb (Thanatos) beinhaltet den Trieb, zu sterben. Beide Triebe sind ständig aktiv und streben nach einer fortwährenden Befriedigung. Denn das Ziel der fanatischen Verhaltensweisen, d. h. der Aggressionen, ist die Vereinzelung, die Verselbständigung und die Individualisierung, Diese Triebe entwickeln ihre eigenen Kräfte: Die Kraft des Eros ist die Kraftäußerung (Triebenergie) der Libido. Die Libido richtet sich auf das Lustvolle im Leben. Besonders geht Freud in diesem Kontext auf die Sexualität ein, die seiner Ansicht nach einen Großteil des menschlichen Strebens darstellt. Die Kraft des Destrudo ist die Kraftäußerung des Thanatos. Das Bündeln dieser psychischen Energien und Kräfte auf Objekte oder Personen nennt Freud Kathexis.

Laplanche, Jean und J. B. Pontalis: Das Vokabular der Psychoanalyse, Suhrkamp, Frankfurt/Main, 1972 S. 147 Titel der Originalausgabe: Vocabulaire de la Psychanalyse, 1967

Jean Laplanche (1924-2012) war ein französischer Theoretiker der Psychoanalyse.

Jean-Bertrand Pontalis (1924 -2013) war ein französischer Philosoph, Psychoanalytiker und Schriftsteller.

25 Bamberger, Richard (et al.), Österreich-Lexikon, Österreichischer Bundesverlag, Wien und München 1968

Richard Bamberger (1911- 2007] war ein österreichischer Literaturforscher.

26 Darüber schrieb Adolf Hitler später in seinem Machwerk Mein Kampf: „Jedenfalls lernte ich langsam den Mann und die Bewegung kennen, die damals Wiens Schicksal bestimmten: Dr. Karl Lueger und die christlich-soziale Partei. Als ich nach Wien kam, stand ich beiden feindselig gegenüber. Der Mann und die Bewegung galten in meinen Augen als, reaktionär'. Das gewöhnliche 
Gerechtigkeitsgefühl aber mußte dieses Urteil in eben dem Maße abändern, in dem ich Gelegenheit erhielt, Mann und Werk kennenzulernen; und langsam wuchs die gerechte Beurteilung zur unverhohlenen Bewunderung. Heute sehe ich in dem Manne mehr noch als früher den gewaltigsten deutschen Bürgermeister aller Zeiten.“ Hitler, Adolf Hitler: Mein Kampf. Zentralverlag der NSDAP (Franz Eher Nachfolger), München, 1925, S. 54-65

Seit 1945 hat der Freistaat Bayern, als Rechtsnachfolger des damaligen Verlages, die Veröffentlichung des Machwerks Mein Kampf in Deutschland verboten. Aber am Jahresende 2015 erlosch das Urheberrecht an diesem Buch, da die gesetzliche Frist 70 Jahre nach dem Tod Hitlers abgelaufen ist.

27 a. a. O., S. 73.

28 LTI S. 343.

29 a. a. O., S. 295.

30 Zweig, Stefan: Schachnovelle, Fischer Taschenbuch Verlag, Frankfurt am Main, 1974, Seite 54.

31 Das Wort Monarchie stammt aus der altgriechischen Sprache und bedeutet ,Alleinherrschaft‘. Der Begriff bezeichnet eine Staats- und / oder Herrschaftsform, bei der ein Adliger zum Staatsoberhaupts auf Lebenszeit, oder bis zu seiner evtl. Abdankung berufen wird. Später kann er, z. B. nach seinem Tod, dieses Amt durch Vererbung an seinen Erben weitergeben.

LTI S. 207.

LTI S. $174 \mathrm{ff}$.

Dieser Ausspruch ist eine Reminiszenz an die Schriftstellerin und Verlegerin Toni Schwabe (1877-1951) und deren Goethe-Roman Der Ausbruch ins Grenzenlose. Schwabe beschäftigte sich in einer Reihe von Romanen mit den Beziehungen von Johann Wolfgang von Goethe und dessen Umgang mit den Frauen in seinem Leben, wobei in diesen Romanen die persönliche Entwicklung der Frauen im Fokus steht. So auch z. B. der 1926 erschienene Roman Der Ausbruch ins Grenzenlose.

36 Joseph Arthur de Gobineau (1816-1882) war ein französischer Diplomat und Schriftsteller. Seine allgemeine Bekanntheit verdankt er dem Versuch über die Ungleichheit der Menschenrassen (Essai sur l'inégalité des races humaines, 18531855). Er gilt deswegen als einer der Begründer des rassistischen Denkens.

\section{LTI S. 183.}

38 Als Quelle gab Wilhelm Scherer Publius Cornelius Tacitus (ca.58 - ca. 120) und dessen Werk Germania an, das im Reclam Verlag, damals noch Leipzig, heute in Ditzingen, 1982, erschienen ist, S. 79 und dort in Randnummer 24.

Scherer, Wilhelm: Geschichte der deutschen Literatur, Th. Knaur Nachf., Berlin, 1930

39 LTI S. 182.

40 Ebd. S. 182.

41 a. a. O., S. 190.

42 Hesse, Max René: Partenau, Rütten \& Loening Verlag, 1929, deutsche Ausgabe, zitiert in LTI S. 38ff.

Inhalt: Geschildert wird in diesem Roman die Unzufriedenheit der Wehrmachtsoffiziere nach dem Ersten Weltkrieg während der Weimarer Republik. Denn die ehemaligen Wehrmachtsoffiziere und Frontkämpfer träumen romantisierend von der Revision des Versailler Vertrags und entwickeln Strategien 
für eine Wiederbewaffnung Deutschlands. In ihrer romantischen Wunschvorstellung sehnen sie sich nach einem starken Mann, einem heilbringenden Führer. Ihr Denkmodell kann als kugelartig beschrieben werden, denn es ist einzig an dem soldatischen Ehren-Codex ausgerichtet und ergo antidemokratisch und antibürgerlich. Für sie existieren Freundschaft und auch Liebe nur innerhalb ihrer militärischen Kaste. Deshalb begeht Oberleutnant Partenau, die zentrale Romanfigur, Selbstmord, als er vor die Entscheidung gestellt wird, sein homoerotisches Verhältnis zu dem Oberfähnrich Kiebold zu lösen.

43 Friedrich Martin von Bodenstedt (1819-1892) war ein deutscher Schriftsteller

44 Klemperer, Victor, LTI: Notizbuch eines Philologen, Reclam, Philipp, jun. Verlag, Ditzingen, 2009 S. 194.

45 Zweig, Stefan: Die Welt von Gestern -Erinnerungen eines Europäers, Anaconda Verlag GmbH, Köln S. 445

Am 18. Februar 1934 durchsuchten vier Polizisten sein Haus, da Stefan Zweig denunziert worden war. Man beschuldigte den erklärten Pazifisten, dass sich in seinem Haus Waffen des Republikanischen Schutzbundes befänden.

Stefan Zweig: Die Welt von Gestern,- Erinnerungen eines Europäers, Anaconda Verlag GmbH, Köln S. 444

Der Republikanische Schutzbund war eine paramilitärische Organisation der österreichischen Sozialdemokratischen Arbeiterpartei (SDAP).

$46 \quad$ LTI S. 453.

47 LTI S. 193

48 Alfred Ernst Rosenberg (1892-1946) war schon zur Zeit der Weimarer Republik (die die Nazis Systemzeit nannten) und des Nationalsozialismus ein führender Ideologe der NSDAP. Im Nürnberger Prozess wurde er wegen Verbrechen an der Menschlichkeit zur Todesstrafe verurteilt und wie andere NS-Verbrecher hingerichtet.

49 LTI S. 135.

50 Die Salzburger Bücherverbrennung fand am 30. April 1938 statt. Aber anders als bei den Bücherverbrennungen 1933 im Deutschen Reich, legten die Verantwortlichen in Salzburg den Schwerpunkt nicht nur auf die Literatur jüdischer Autoren, sondern auch auf die der Auslöschung der katholischen, stände-staatlichen bzw. austrofaschistischen und dem österreichischen Kaiserhaus nahestehenden Bücher.

51 Zweig, Stefan: Schachnovelle, Fischer Taschenbuch Verlag, Frankfurt am Main, 1974, S. 20.

52 Flashar, Hellmut (Hrsg.): Aristoteles-Peripatos, Schwabe Verlag, Basel 2004, S. 459f.

Hellmut Flashar (geboren 1929) wirkte als Professor für Klassische Philologie an den Universitäten Bochum und München.

53 Peripatos ist der Name der philosophischen Schule des Aristoteles. Sie erhielt ihren Namen von dem Ort, an dem der Unterricht stattfand, in diesem Fall vom Peripatos für „Wandelhalle“.

Wehrli, Fritz (Hrsg.): Die Schule des Aristoteles. Texte und Kommentare, 10 Hefte, Schwabe Verlag Basel (1967-1969) sowie dazu erschienen 2 Supplemente. Ebenda 1974

54 Zweig, Stefan: Schachnovelle, Fischer Taschenbuch Verlag, Frankfurt am Main, 1974, S. 23. 
55 Mit „ein Gall“ ist, nach dessen Biographen Schramm-Macdonald, wohl der deutsche Arzt und Anatom Franz Joseph Gall (1758-1828) gemeint. Er flüchtete aus Deutschland und praktizierte in Paris. Gall trug mit seinen Arbeiten maßgeblich zur Lehre der Physiognomie bei. Darin wird dargelegt, wie persönliche Charakterzüge bzw. Eigenschaften im Gesicht eines Menschen, d. h. in seiner Mimik, ablesbar sind. Zudem entdeckte er auch die Fasermuskelstruktur des Gehirns, die Stefan Zweig im o.g. Zitat, als ,Schachmuskel oder Schachhöcker “ anzieht.

Schramm-Macdonald: Gall, Franz Joseph. In: Allgemeine Deutsche Biographie $(A D B)$. Band 8, Verlag Duncker \& Humblot, München und Leipzig 1979, S. 315 f.

Karl-Hugo Schramm-Macdonald (1837- 1914) war ein deutscher (Wissenschafts-) Journalist aus Dresden. Er war Mitarbeiter der Allgemeinen Deutschen Biographie, die die Historische Commission bei der königl. Akademie der Wissenschaften herausgab.

56 Die deutschen Autoren Ludwig Klages (1872-1956), Rudolf Kassner (1873- 1959), Max Picard (1888-1965) und der antidemokratische politische Schriftsteller Oswald Spengler (1880-1936) haben bei den gebildeten Leser-Schichten ein physiognomisches Vorbewusstsein entwickelt, das aus Biologie, Phänomenologie und Religion ein ,gesundes Volksempfinden“ machte und das in der Zeit des NSRegimes die missbräuchliche Umschreibung für die angeblich unverderbte Meinung des Volkes wurde.

57 Zweig, Stefan: Schachnovelle, Fischer Taschenbuch Verlag, Frankfurt am Main, 1974, Seite 38.

$58 \quad$ LTI S. 81.

59 a. a. O., S. 125.

60 a. a. O., S. 126.

61 a. a. O., S. 121.

62 „Hestra der Wigru“ bedeutet: Herstellungsanweisung der Wirtschaftsgruppe“.

63 Zweig, Stefan: Schachnovelle, Fischer Taschenbuch Verlag, Frankfurt am Main, 1974, Seite 19.

64 a.a.O. S. 26.

65 Geulen, Christian: Geschichte des Rassismus, C.H. Beck-Verlag, München 2007, S. $97 \mathrm{f}$.

Christian Geulen (geboren 1969) ist ein deutscher Historiker und Hochschullehrer.

66 Aplomb ist eine Substantivierung von à plomb für senkrecht, welches sich aus der Präposition à und dem Substantiv plomb für ein Senkblei (Lot) zusammensetzt. Plomb stammt vom lateinischen plumbum für Blei ab. Also geht das französische aplomb auf eine senkrechte Haltung zurück und ist auch gleichbedeutend mit Gelassenheit. Der Ausdruck Aplomb entstand im 18. Jahrhundert, als das vornehme Benehmen der „höheren“ Gesellschaftsschichten stark von den Tanzbewegungen geprägt war. Damals beschrieb man damit die Fähigkeit zum Abfangen einer Bewegung z. B. beim Ballett. Noch heute spricht man in diesem Sinn, aber auch im übertragenen Sinn von dieBalance halten.

Wissenschaftlicher Rat der Dudenredaktion, Annette Klosa u. a. (Hrsg.): Duden, Deutsches Universalwörterbuch. Dudenverlag, Mannheim/Leipzig/Wien/Zürich 2001, „Aplomb“, S. 156.

67 Zweig, Stefan: Schachnovelle, Fischer Taschenbuch Verlag, Frankfurt am Main, 1974, S. 32. 
68 Im Jahr 1873 grub Heinrich Schliemann, inspiriert durch Homers Epos vom trojanischen Krieg, in der Türkei einen prunkvollen Goldschatz aus der Antike aus und deklarierte ihn als den Goldschatz des Priamos. Aber auch die dabei entdeckte Goldmaske, in der Schliemann den Agamemnon zu erkennen glaubte, stammte nicht aus der Zeit des trojanischen Krieges. Die gefundenen Goldschätze waren Grabbeigaben eines mykenischen Fürsten, der rund 300 Jahre vor dem mythischen König der Griechen lebte. Aber obwohl Schliemann sich bei der Einordnung der Funde irrte, lösten sie im deutschen Sprachraum eine neue Begeisterungswelle für die gesamte griechische Kultur aus.

${ }_{69}$ Zweig, Stefan: Schachnovelle, Fischer Taschenbuch Verlag, Frankfurt am Main, 1974, S. 21.

70 Polybios bezeichnet Tyrannis als die illegitime, entartete, despotische Form der Monarchie, weil sie sich am Eigennutz der Regierenden orientiert. Ähnliche werden die Oligarchie und Ochlokratie als Staatsformen definiert. (Ochlokratie d. h. Pöbelherrschaft). Bei diesen Herrschaftsformen geht die Orientierung am Gemeinwohl verloren, und statt dessen bestimmen Eigennutz und Habsucht das Handeln der Tyrannen). Die am Gemeinwohl orientierten Herrschaftsformen sind z. B. Monarchie, Aristokratie, Demokratie.

71 Zweig, Stefan: Schachnovelle, Fischer Taschenbuch Verlag, Frankfurt am Main, 1974, S. 21.

72 a.a. O. S. 54.

73 Wilhelm Friedrich Boger (1906-1977) war ein deutscher SS-Oberscharführer sowie Mitarbeiter der Politischen Abteilung Referat Flucht, Diebstahl und Fahndung im KZ Auschwitz. Er führte dort eine als Bogerschaukel bezeichnete Foltermethode ein.

74 Langenbein, Hermann: Menschen in Auschwitz, Europa Verlag Hamburg, 1999, S. 433f.

Hermann Langbein (1912-1995) war ein österreichischer kommunistischer Widerstandskämpfer und Historiker. Der ehemalige KZ-Häftling in Auschwitz war 1954 Mitbegründer des Internationalen Auschwitz Komitees. 